\title{
THE THEORY OF JACOBI FORMS OVER THE CAYLEY NUMBERS
}

\author{
M. EIE AND A. KRIEG
}

\begin{abstract}
As a generalization of the classical theory of Jacobi forms we discuss Jacobi forms on $\mathscr{H} \times \mathbb{C}^{8}$, which are related with integral Cayley numbers. Using the Selberg trace formula we give a simple explicit formula for the dimension of the space of Jacobi forms. The orthogonal complement of the space of cusp forms is shown to be spanned by certain types of Eisenstein series.
\end{abstract}

\section{INTRODUCTION}

The classical theory of Jacobi forms on $H \times \mathbb{C}$ was described by Eichler and Zagier [4] in 1985. There also exist more general types of Jacobi forms on $H \times$ $\mathbb{C}^{n}$ considered by Gritsenko [8] or for the Siegel half-space considered by Ziegler [14]. These Jacobi forms naturally appear in the Fourier-Jacobi expansion of Siegel modular forms (cf. [12]).

Jacobi forms over the Cayley numbers are defined on $H \times \mathbb{C}^{8}$. They were introduced in [5 and 6], where they appeared as Fourier-Jacobi coefficients of modular forms on the half-plane of the Cayley numbers of degree 2. They are of special interest, since they are related with modular forms on the exceptional domain (cf. [1,9]). On the other hand, the arithmetic of integral Cayley numbers (cf. [2]) leads to special results, which cannot be obtained in the general case.

In this paper we show that the space of Jacobi forms over the Cayley numbers has finite dimension. We can demonstrate that the orthogonal complement of the space of cusp forms is spanned by certain Eisenstein series. Moreover the Selberg trace formula can be applied in order to determine the dimension of the space of Jacobi cusp forms explicitly. This leads to a very simple dimension formula involving a weighted summatory function of Euler's totient function $\varphi(n)$. Surprisingly the result is simpler than in the classical situation (cf. [4]) or for Jacobi forms of index 1 on $H \times \mathbb{C}^{n}$ (cf. [13]).

Received by the editors July 13, 1992.

1991 Mathematics Subject Classification. Primary 11F55, 11 F72.

Key words and phrases. Jacobi form, Eisenstein series, theta series, vector valued elliptic modular form, Selberg trace formula.

The first author's work was supported by the National Science Foundation of Taiwan, Republic of China (NSC-810298M194504). 


\section{Notations}

Let $\mathfrak{f}$ be a field. The set $\mathscr{C}=\mathscr{C}_{\mathfrak{f}}$ of the Cayley numbers over $\mathfrak{f}$ is an eight dimensional vector space over $f$ with the standard basis $e_{0}, e_{1}, e_{2}, e_{3}, e_{4}, e_{5}, e_{6}$, $e_{7}$ satisfying the following rules for multiplication:

$$
\begin{gathered}
x e_{0}=e_{0} x=x \text { for all } x \in \mathscr{C}, \\
e_{i}^{2}=-e_{0}, \quad i=1,2,3,4,5,6,7, \\
e_{1} e_{2} e_{4}=e_{2} e_{3} e_{5}=e_{3} e_{4} e_{6}=e_{4} e_{5} e_{7}=e_{5} e_{6} e_{1}=e_{6} e_{7} e_{2}=e_{7} e_{1} e_{3}=-e_{0} .
\end{gathered}
$$

Write $x \in \mathscr{C}$ in the form $x=\sum_{j=0}^{7} x_{j} e_{j}, x_{j} \in \mathfrak{f}$. Then we consider the following mappings:

(a) involution: $\mathscr{C} \rightarrow \mathscr{C}, x \mapsto \bar{x}=2 x_{0} e_{0}-x=x_{0} e_{0}-\sum_{j=1}^{7} x_{j} e_{j}$,

(b) norm: $\mathscr{C} \rightarrow \mathfrak{f}, N(x)=x \bar{x}=\sum_{j=0}^{7} x_{j}^{2}$,

(c) bilinear form: $\mathscr{C} \times \mathscr{C} \rightarrow \mathfrak{f}, \sigma(x, y)=2 \sum_{j=0}^{7} x_{j} y_{j}$, if $y=\sum_{j=0}^{7} y_{j} e_{j}$.

In particular, one has

$$
N(x+y)=N(x)+N(y)+\sigma(x, y) \text { for all } x, y \in \mathscr{C} .
$$

Cf. [3, Chapter 9], for further details.

Let $a \subset \mathscr{C}_{\mathbb{Q}}$ be the $\mathbb{Z}$-module of integral Cayley numbers investigated by Coxeter [2]. A basis of $a$ is given by $\alpha_{0}, \ldots, \alpha_{7}$ where

$$
\begin{gathered}
\alpha_{0}=e_{0}, \quad \alpha_{1}=e_{1}, \quad \alpha_{2}=e_{2}, \quad \alpha_{3}=e_{4}, \\
\alpha_{4}=\frac{1}{2}\left(e_{1}+e_{2}+e_{3}-e_{4}\right), \quad \alpha_{5}=\frac{1}{2}\left(-e_{0}-e_{1}-e_{4}+e_{5}\right), \\
\alpha_{6}=\frac{1}{2}\left(-e_{0}+e_{1}-e_{2}+e_{6}\right), \quad \alpha_{7}=\frac{1}{2}\left(-e_{0}+e_{2}+e_{4}+e_{7}\right) .
\end{gathered}
$$

We can identify $\mathscr{C}_{\mathbb{C}}$ with $\mathbb{C}^{8}$ via the standard basis $e_{0}, \ldots, e_{7}$. Let $H$ stand for the upper half-plane in $\mathbb{C}$,

$$
H=\{z \in \mathbb{C} \mid z=x+i y, y>0\} .
$$

Let $k, m$ be integers and $m \geq 0$. A holomorphic function $f: H \times \mathscr{C}_{\mathbb{C}} \rightarrow \mathbb{C}$ is called a Jacobi form of weight $k$ and index $m$, if it satisfies the following conditions:

(j.1)

$$
\begin{aligned}
& f(z, w)=\left.f\right|_{k, m}[M](z, w) \\
& :=(c z+d)^{-k} e^{-2 \pi i m c N(w) /(c z+d)} f\left(\frac{a z+b}{c z+d}, \frac{w}{c z+d}\right) \\
& \quad \text { for all } M=\left(\begin{array}{ll}
a & b \\
c & d
\end{array}\right) \in \Gamma:=\mathrm{SL}_{2}(\mathbb{Z}) .
\end{aligned}
$$

$$
\begin{aligned}
f(z, w)=\left.f\right|_{m}[\lambda, \mu](z, w):=e^{2 \pi i m[N(\lambda) z+\sigma(\lambda, w)]} f(z, w+\lambda z+\mu) \\
\quad \text { for all } \lambda, \mu \in a .
\end{aligned}
$$

(j.3) $f$ has a Fourier expansion of the form

$$
f(z, w)=\sum_{n=0}^{\infty} \sum_{t \in o, n m \geq N(t)} \alpha_{f}(n, t) e^{2 \pi i[n z+\sigma(t, w)]}
$$


$f$ is a Jacobi cusp form, if it moreover satisfies

(j.4) $\alpha_{f}(n, t)=0$, whenever $n m=N(t)$.

We denote by $J_{k, m}(a)$ (resp. $\left.J_{k, m}^{0}(a)\right)$ the space of Jacobi forms (resp. Jacobi cusp forms) over the Cayley numbers of weight $k$ and index $m$. Examples of functions in $J_{k, m}(a)$ are given by the Fourier-Jacobi coefficients of modular forms on the half-plane of the Cayley numbers of degree 2 (cf. [6]).

Given a congruence subgroup $\Gamma^{\prime}$ of $\Gamma=\mathrm{SL}_{2}(\mathbb{Z})$ let $M_{k}\left(\Gamma^{\prime}\right)$ (resp. $\left.S_{k}\left(\Gamma^{\prime}\right)\right)$ denote the space of entire modular forms (resp. cusp forms) of weight $k$ with respect to $\Gamma^{\prime}$ (cf. [11]). Using (j.3) and (j.1) we get

$$
J_{k, 0}(a) \cong M_{k}(\Gamma), \quad J_{k, 0}^{0}(a) \cong S_{k}(\Gamma) .
$$

Therefore we will always assume $m \geq 1$. It follows from [6] that

$$
J_{k, 1}(a) \cong M_{k-4}(\Gamma), \quad J_{k, 1}^{0}(a) \cong S_{k-4}(\Gamma) \quad \text { for even } k>0 .
$$

\section{THETA SERIES}

Given $m \geq 1$ and $q \in a$ we define the theta series

$$
\vartheta_{m, q}(z, w)=\sum_{\nu \in \Lambda(q)} e^{2 \pi i m[N(\nu) z+\sigma(\nu, w)]},
$$

where $\Lambda(q):=\{t+q / m \mid t \in o\}$. Then $\vartheta_{m, 0} \in J_{4,1}$ was shown in [6]. Due to (1) an easy calculation yields

$$
\begin{gathered}
\left.\vartheta_{m, q}\right|_{m}[\lambda, \mu]=\vartheta_{m, q} \text { for all } \lambda, \mu \in o, \\
\vartheta_{m, q}(z+1, w)=e^{2 \pi i N(q) / m} \vartheta_{m, q}(z, w) .
\end{gathered}
$$

Given $w \in \mathscr{C}_{\mathbb{C}}$ we define

$$
\hat{w}=\left(w_{0}, \ldots, w_{7}\right)^{t} \in \mathbb{C}^{8}, \quad \text { whenever } w=\sum_{j=0}^{7} w_{j} \alpha_{j} .
$$

The $8 \times 8$ matrix $S=\left(\sigma\left(\alpha_{i}, \alpha_{j}\right)\right)$ is positive definite, even and unimodular (cf. [6]). In the notation of [10, p. 101], we therefore have

$$
\vartheta_{m, q}(z, w)=\boldsymbol{\Theta}_{\hat{q}, m S \hat{w} / m}\left(z, m S ; \mathbb{Z}^{8}\right) .
$$

Using [10, IV.2.3], we obtain

$$
\begin{aligned}
\left.\vartheta_{m, q}\right|_{4, m}[J](z, w)=z^{-4} e^{-\pi i m S[\hat{w}] / z} \boldsymbol{\Theta}_{\hat{q} / m, m S \hat{w} / z}\left(-1 / z, m S ; \mathbb{Z}^{8}\right) \\
\quad=m^{-4} e^{-\pi i m S[\hat{w}] / z+2 \pi i \hat{q}^{t} S \hat{w} / z} \boldsymbol{\Theta}_{m S \hat{w} / z,-\hat{q} / m}\left(z,(m S)^{-1} ; \mathbb{Z}^{8}\right) \\
=m^{-4} \sum_{g \in \mathbb{Z}^{8}} e^{\pi i z(m S)^{-1}[g]+2 \pi i g^{t} \hat{w}-2 \pi i g^{t} \hat{q} / m} .
\end{aligned}
$$

Here $J=\left(\begin{array}{cc}0 & -1 \\ 1 & 0\end{array}\right)$. If one sets $g=m S(h+\hat{p} / m), h \in \mathbb{Z}^{8}, p: o / m_{\circ}$, the result is

$$
\left.\vartheta_{m, q}\right|_{4, m}[J](z, w)=m^{-4} \sum_{p: o / m o} e^{-2 \pi i \sigma(q, p) / m} \vartheta_{m, p}(z, w) .
$$

Next a combination of [10, IV.3.6 and IV.1.3], yields

(8) $\left.\vartheta_{m, q}\right|_{4, m}[M]=\vartheta_{m, q}, \quad$ whenever $M \in \Gamma, M \equiv\left(\begin{array}{ll}1 & 0 \\ 0 & 1\end{array}\right)(\bmod m)$.

Let $U(n)$ denote the unitary group contained in $\mathrm{GL}_{n}(\mathbb{C})$. We fix a set of representatives $q_{1}, \ldots, q_{m^{8}}$ of $o / m_{0}$ and set

$$
\boldsymbol{\Theta}:=\left(\vartheta_{m, q_{1}}, \ldots, \vartheta_{m, q_{m}}\right)^{t} \text {. }
$$


The operations in (j.1) and (j.2) can be applied to each component of $\boldsymbol{\theta}$.

Proposition 1. There exists a unique homomorphism of the groups $\psi: \Gamma \rightarrow$ $U\left(m^{8}\right)$ such that

$$
\left.\Theta\right|_{4, m}[M]=\overline{\psi(M)} \cdot \Theta \text { for all } M \in \Gamma .
$$

The principal congruence subgroup

$$
\Gamma[m]=\left\{M \in \Gamma \mid M \equiv\left(\begin{array}{ll}
1 & 0 \\
0 & 1
\end{array}\right)(\bmod m)\right\}
$$

is contained in the kernel of $\psi$.

Proof. According to the uniqueness of the Fourier expansion with respect to $w$ in (4), the $m^{8}$ components of $\Theta$ are linearly independent functions. This implies the uniqueness of $\psi$. It suffices to demonstrate (10) for generators of $\Gamma$. Thus (10) follows from (6) and (7). The last statement is a consequence of (8).

In particular one has

$$
\begin{array}{cc}
\psi(T)=\operatorname{diag}\left(e^{-2 \pi i N\left(q_{1}\right) / m}, \ldots, e^{-2 \pi i N\left(q_{m} 8\right) / m}\right), & T=\left(\begin{array}{cc}
1 & 1 \\
0 & 1
\end{array}\right) \\
\psi(J)=\overline{\psi(-J)} t=\left(m^{-4} e^{2 \pi i \sigma\left(q_{\nu}, q_{\mu}\right) / m}\right)_{\nu, \mu=1, \ldots, m^{8},} & J=\left(\begin{array}{cc}
0 & -1 \\
1 & 0
\end{array}\right),
\end{array}
$$

according to (6) and (7).

Let $f \in J_{k, m}(a)$ with the Fourier expansion (j.3). Given $q \in a$ we set

$$
F_{q}(z):=\sum_{n \in \mathbb{N}_{0}, n \geq N(q) / m} \alpha_{f}(n, q) e^{2 \pi i[n-N(q) / m] z} .
$$

Proposition 2. Given $f \in J_{k, m}(a)$ one has a unique representation

$$
f(z, w)=\sum_{q: o / m o} F_{q}(z) \cdot \vartheta_{m, q}(z, w) .
$$

Proof. Using (j.2) we have

$$
\begin{aligned}
f(z, w) & =e^{2 \pi i m[N(\lambda) z+\sigma(\lambda, w)]} f(z, w+\lambda z) \\
& =\sum_{t \in o} \sum_{n \geq N(t) / m} \alpha_{f}(n, t) e^{2 \pi i[(n+\sigma(t, \lambda)+m N(\lambda)) z+\sigma(t+m \lambda, w)]}
\end{aligned}
$$

for all $\lambda \in a$. Comparing the coefficients we get

$$
\alpha_{f}(n+\sigma(t, \lambda)+m N(\lambda), t+m \lambda)=\alpha_{f}(n, t) \text { for all } n, t, \lambda .
$$

Hence we have $F_{q+m \lambda}=F_{q}$ in (13). Thus the right-hand side of (14) is well defined. Setting $t=m \lambda+q, \lambda \in o, q: o / m_{o}$, a rearrangement of the Fourier expansion of $f$ yields (14).

Just as in (9), now set

$$
F:=\left(F_{m, q_{1}}, \ldots, F_{m, q_{m 8}}\right)^{t} .
$$

Given a function $\Phi: H \rightarrow \mathbb{C}$, set

$$
\left.\Phi\right|_{k} M(z):=(c z+d)^{-k} \Phi\left(\frac{a z+b}{c z+d}\right) \quad \text { for } M=\left(\begin{array}{ll}
a & b \\
c & d
\end{array}\right) \in \Gamma .
$$

Apply this definition to each component of $F$. 
Theorem 1. Given $k, m \in \mathbb{Z}, m \geq 1$, the mappings

$$
\begin{array}{r}
\left\{F \in M_{k-4}(\Gamma[m])^{m^{8}}|F|_{k-4} M=\psi(M) \cdot F \text { for all } M \in \Gamma\right\} \rightarrow J_{k, m}(0), \\
F \mapsto F^{t} \cdot \boldsymbol{\Theta},
\end{array}
$$

as well as

$$
\begin{aligned}
\left\{F \in S_{k-4}(\Gamma[m])^{m^{8}}|F|_{k-4} M=\psi(M) \cdot F \text { for all } M \in \Gamma\right\} \rightarrow J_{k, m}^{0}(a), & \\
\qquad & F F^{t} \cdot \boldsymbol{\Theta},
\end{aligned}
$$

are isomorphisms.

Proof. Given $f \in J_{k, m}(a)$, apply (14) and Proposition 1,

$$
\begin{aligned}
F^{t} \cdot \Theta & =f=\left.f\right|_{k, m}[M]=\left(\left.F\right|_{k-4} M\right)^{t} \cdot\left(\left.\Theta\right|_{4, m}[M]\right) \\
& \left.=\left.(\overline{\psi(M)})^{t} F\right|_{k-4} M\right)^{t} \cdot \Theta .
\end{aligned}
$$

Since the components of theta are linearly independent and $\psi(M)$ is unitary, we get

$$
\left.F\right|_{k-4} M=\psi(M) \cdot F \text { for all } M \in \Gamma \text {. }
$$

Proposition 1 leads to $F_{q} \in M_{k-4}(\Gamma[m])$ for each $q \in 0$. Comparing the Fourier expansions we conclude that $f$ is a cusp form if and only if each $F_{q}$, $q \in a$, is a cusp form.

Starting with $F \in M_{k-4}(\Gamma[m])^{m^{8}}$ such that $\left.F\right|_{k-4} M=\psi(M) \cdot F$, we obtain $\left.f\right|_{k, m}[M]=f$ for $f=F^{t} \cdot \boldsymbol{\Theta}$ and $M \in \Gamma$ from Propositions 1 and 2. Finally (5) yields (j.2). Since (j.3) is clear, we get $f \in J_{k, m}(o)$.

As an immediate consequence of Theorem 1 we obtain

Corollary 1. Given $k, m \in \mathbb{Z}, m \geq 1$, one has

$$
\operatorname{dim} J_{k, m}(a) \leq m^{8} \cdot \operatorname{dim} M_{k-4}(\Gamma[m])<\infty,
$$

in particular,

$$
\begin{gathered}
\operatorname{dim} J_{k, 1}(0)=\operatorname{dim} M_{k-4}(\Gamma), \quad \operatorname{dim} J_{k, 1}^{0}(a)=\operatorname{dim} S_{k-4}(\Gamma), \\
J_{k, m}(a)=\{0\}, \quad \text { if } k<4 .
\end{gathered}
$$

\section{EISENSTEIN SERIES}

Let $\Gamma_{\infty}=\left\{\left(\begin{array}{ll}1 & n \\ 0 & 1\end{array}\right) \mid n \in \mathbb{Z}\right\}$. Given $q \in a$ with $N(q) \equiv 0(\bmod m)$ we define the Jacobi-Eisenstein series

(16)

$$
\begin{aligned}
& E_{k, m}(z, w ; q) \\
& \begin{array}{l}
:=\frac{1}{2} \sum_{M: \Gamma_{\infty} \backslash \Gamma}(c z+d)^{-k} \sum_{\lambda \in \Lambda(q)} \exp \left\{2 \pi i m \left[N(\lambda) \frac{a z+b}{c z+d}\right.\right. \\
\left.\left.\quad+\sigma\left(\lambda, \frac{w}{c z+d}\right)-\frac{c N(w)}{c z+d}\right]\right\} \\
=\left.\left.\frac{1}{2} \sum_{M: \Gamma_{\infty} \backslash \Gamma} \sum_{\lambda \in \Lambda(q)} 1\right|_{m}[\lambda, 0]\right|_{k, m}[M](z, w),
\end{array}
\end{aligned}
$$


where $M=\left(\begin{array}{ll}a & b \\ c & d\end{array}\right)$ and $\Lambda(q)=\{t+q / m \mid t \in o\}$. Due to $m N(\lambda) \in \mathbb{N}_{0}$ for all $\lambda \in \Lambda(q)$, the definition does not depend on the choice of representatives of $\Gamma_{\infty} \backslash \Gamma$. It is obvious that

(17) $E_{k, m}(z, w ; q)=E_{k, m}\left(z, w ; q^{\prime}\right), \quad$ if $q=q^{\prime}+m \lambda$ for some $\lambda \in o$,

$$
E_{k, m}(z, w ;-q)=E_{k, m}(z,-w ; q)=(-1)^{k} E_{k, m}(z, w ; q) \text {. }
$$

Proposition 3. Let $k, m \in \mathbb{N}, k>10$ and $q \in a$ with $N(q) \equiv 0(\bmod m)$. Then the series (16) converges absolutely and locally uniformly in $H \times \mathscr{E}_{\mathbb{C}}$.

Proof. It is well known from the theta transformation formula that

$$
\sum_{n \in \mathbb{Z}} e^{-\pi n^{2} y}=O\left(1+y^{-1 / 2}\right) \text { for } y>0 .
$$

Hence we obtain

$$
\sum_{\lambda \in \frac{1}{m^{o}}} e^{-2 \pi m y N(\lambda)}=O\left(1+y^{-4}\right) \text { for } y>0 .
$$

Given $(z, w)$ in a compact subset of $H \times \mathscr{C}_{\mathbb{C}}$ we get

$$
\begin{aligned}
& \sum_{\lambda \in \Lambda(q)}\left|\exp \left\{2 \pi i m\left[N(\lambda) \frac{a z+b}{c z+d}+\sigma\left(\lambda, \frac{w}{c z+d}\right)-\frac{c N(w)}{c z+d}\right]\right\}\right| \\
& \quad \leq \gamma \sum_{\lambda \in \frac{1}{m^{o}}} \exp \left\{-2 \pi m\left[\left(\operatorname{Im} \frac{a z+b}{c z+d}\right) N(\lambda)-\delta \sqrt{N(\lambda)} \sqrt{\operatorname{Im} \frac{a z+b}{c z+d}}\right]\right\},
\end{aligned}
$$

where the positive constants $\gamma$ and $\delta$ only depend on the compact set. There exists a constant $\varepsilon>0$ such that

$$
\varepsilon \operatorname{Im} \frac{a i+b}{c i+d} \leq \operatorname{Im} \frac{a z+b}{c z+d} \leq \varepsilon^{-1} \operatorname{Im} \frac{a i+b}{c i+d}
$$

holds for all $\left(\begin{array}{ll}a & b \\ c & d\end{array}\right) \in \Gamma$ and $z$ in the compact set. Hence the above sum over $\lambda$ is uniformly majorized by

$$
\sum_{\lambda \in \frac{1}{m} \mathcal{O}} \exp \left\{-\pi m\left(\operatorname{Im} \frac{a i+b}{c i+d}\right) N(\lambda)\right\}=O\left(1+\left[\operatorname{Im} \frac{a i+b}{c i+d}\right]^{-4}\right) .
$$

Since the series $\sum_{M}: \Gamma_{\infty}|\Gamma| c i+\left.d\right|^{8-k}$ converges for $k>10$, the series (16) converges and defines a holomorphic function on $H \times \mathscr{b}_{\mathbb{C}}$.

From the group property of $\Gamma=\mathrm{SL}_{2}(\mathbb{Z})$ and (16), it is easy to verify that $E_{k, m}(z, w ; q)$ satisfies (j.1). Given $\lambda, \mu \in \sigma$ we obtain

$$
\begin{gathered}
\left.E_{k, m}(\cdot, \cdot ; q)\right|_{m}[\lambda, \mu](z, w)=\frac{1}{2} \sum_{M: \Gamma_{\infty} \backslash \Gamma}(c z+d)^{-k} \\
\cdot \sum_{\nu \in \Lambda(q)} \exp \left\{2 \pi i m \left[N(\nu) \frac{a z+b}{c z+d}+\sigma\left(\nu, \frac{w+\lambda z+\mu}{c z+d}\right)\right.\right. \\
\left.\left.-\frac{c N(w+\lambda z+\mu)}{c z+d}+N(\lambda) z+\sigma(\lambda, w)\right]\right\} .
\end{gathered}
$$


Next a simple calculation leads to

$$
\begin{array}{r}
N(\nu) \frac{a z+b}{c z+d}+\sigma\left(\nu, \frac{w+\lambda z+\mu}{c z+d}\right)-\frac{c N(w+\lambda z+\mu)}{c z+d}+N(\lambda) z+\sigma(\lambda, w) \\
=N(\nu+d \lambda-c \mu) \frac{a z+b}{c z+d}+\sigma\left(\nu+d \lambda-c \mu, \frac{w}{c z+d}\right)-\frac{c N(w)}{c z+d}+\alpha
\end{array}
$$

where $\alpha=\sigma(\nu, a \mu-b \lambda)+b c \cdot \sigma(\lambda, \mu)-b d \cdot N(\lambda)-a c \cdot N(\mu) \in \frac{1}{m} \mathbb{Z}$. Hence a rearrangement yields (j.2) for $E_{k, m}(z, w ; q)$.

Theorem 2. Let $k, m \in \mathbb{N}, k>10$ and $q \in 0$ with $N(q) \equiv 0(\bmod m)$. Then the Jacobi-Eisenstein series $E_{k, m}(z, w ; q)$ belongs to $J_{k, m}(a)$ and possesses $a$ Fourier expansion of the form

$$
\begin{aligned}
& E_{k, m}(z, w ; q)=\frac{1}{2}\left(\vartheta_{m, q}(z, w)+(-1)^{k} \vartheta_{m,-q}(z, w)\right) \\
& +\sum_{n=1}^{\infty} \sum_{t \in o, n m>N(t)} \alpha_{q}(n, t) e^{2 \pi i[n z+\sigma(t, w)]}
\end{aligned}
$$

Proof. Convergence and analyticity were proved in Proposition 3. (j.1) and (j.2) were shown above.

Choosing $c=0$ the sum over $\lambda \in \Lambda(q)$ as well as $d= \pm 1$ in (16) exactly yields $\vartheta_{m, q}(z, w)+(-1)^{k} \vartheta_{m,-q}(z, w)$. Now let $c \neq 0$. Setting $\lambda=\frac{q}{m}+p+c t$, $p: a / c a, t \in a$, we get

$$
\begin{aligned}
\sum_{\lambda \in \Lambda(q)} & \exp \left\{2 \pi i m\left[N(\lambda) \frac{a z+b}{c z+d}+\sigma\left(\lambda, \frac{w}{c z+d}\right)-\frac{c N(w)}{c z+d}\right]\right\} \\
= & \sum_{p: o / c_{o}} \exp \left\{\frac{2 \pi i a N(q+m p)}{m c}\right\} \\
& \times \sum_{t \in o} \exp \left\{-2 \pi i m\left[N\left(w-\frac{q}{m c}-\frac{p}{c}-t\right) /(z+d / c)\right]\right\} \\
= & \left(\frac{z+d / c}{m}\right)^{4} \sum_{p: o / c o} \exp \left\{\frac{2 \pi i a N(q+m p)}{m c}\right\} \\
& \times \sum_{t \in o} \exp \left\{2 \pi i\left[N(t) \frac{z+d / c}{m}+\sigma\left(w-\frac{q}{m c}-\frac{p}{c}, t\right)\right]\right\}
\end{aligned}
$$

where we applied the theta transformation formula [10, IV.2.2], just as in $\S 2$. For fixed $0 \neq c \in \mathbb{Z}$ we now sum over $d=d^{\prime}+m c l, 1 \leq d^{\prime} \leq m|c|,\left(c, d^{\prime}\right)=$ $1, l \in \mathbb{Z}$. In view of the well-known identity

$$
\sum_{l \in \mathbb{Z}}\left(\frac{z}{m}+\frac{d^{\prime}}{m c}+l\right)^{4-k}=\frac{(-2 \pi i)^{k-4}}{(k-5) !} \sum_{n=1}^{\infty} n^{k-5} \exp \left\{2 \pi i n\left(\frac{z}{m}+\frac{d^{\prime}}{m c}\right)\right\}
$$


we obtain a Fourier expansion of the form

$$
\begin{aligned}
\frac{1}{2} \sum_{M: \Gamma_{\infty} \backslash \Gamma, c \neq 0}(c z+d)^{-k} \sum_{\lambda \in \Lambda(q)} \exp \left\{2 \pi i m \left[N(\lambda) \frac{a z+b}{c z+d}\right.\right. & \left.\left.+\sigma\left(\lambda, \frac{w}{c z+d}\right)-\frac{c N(w)}{c z+d}\right]\right\} \\
= & \sum_{n=1}^{\infty} \sum_{t \in o} \alpha(n, t) e^{2 \pi i[z(n+N(t)) / m+\sigma(t, w)]} .
\end{aligned}
$$

Since $E_{k, m}(z, w ; q)$ and $\vartheta_{m, q}(z, w)+(-1)^{k} \vartheta_{m,-q}(z, w)$ are invariant under $z \mapsto z+1$, we conclude $\alpha(n, t)=0$ unless $n+N(t) \equiv 0(\bmod m)$. This gives (19).

Remark 1. Let $k, m \in \mathbb{N}, k>10$ and $q \in 0$ with $N(q) \equiv 0(\bmod m)$. A look at (17), (18) and at the Fourier expansion (19) yields

$$
E_{k, m}(\cdot, \cdot ; q) \equiv 0 \text { if and only if } k \text { is odd and } 2 q \in m_{0} \text {. }
$$

Moreover fix representatives $\pm q_{1}, \ldots, \pm q_{r}, q_{r+1}, \ldots, q_{r+s}$ of $q: a / m_{0}$ with $N(q) \equiv 0(\bmod m)$ such that $2 q_{j} \notin m_{\circ}$ for $1 \leq j \leq r$ and $2 q_{j} \in m_{0}$ for $r<j \leq r+s$. Define

$$
\begin{gathered}
\mathscr{E}_{k, m}:=\left\{E_{k, m}\left(\cdot, \cdot ; q_{j}\right) \mid 1 \leq j \leq r\right\}, \quad \text { if } k \text { is odd, } \\
\mathscr{E}_{k, m}:=\left\{E_{k, m}\left(\cdot, \cdot ; q_{j}\right) \mid 1 \leq j \leq r+s\right\}, \quad \text { if } k \text { is even } .
\end{gathered}
$$

Then (19) and the linear independence of the theta series imply that the set $\mathscr{E}_{k, m}$ is linearly independent.

Now let $m=1$ and therefore $q=0$ and $k$ be even. Then our proof describes the Fourier development explicitly. If $\sigma_{k}$ denotes the divisor sum, $B_{k}$ the Bernoulli number and $E_{k}(z) \in M_{k}(\Gamma)$ the normalized Eisenstein series (cf. [11]), we obtain

Corollary 2. Let $k>10$ be even. Then the Fourier expansion of the JacobiEisenstein series of weight $k$ and index 1 is given by

$$
E_{k, 1}(z, w ; 0)=\sum_{n=0}^{\infty} \sum_{t \in \sigma, N(t) \leq n} \gamma(n, t) e^{2 \pi i[n z+\sigma(z, w)]},
$$

where

One has

$$
\gamma(n, t)= \begin{cases}1 & \text { if } n=N(t), \\ -\frac{2(k-4)}{B_{k-4}} \sigma_{k-5}(n-N(t)) & \text { if } n>N(t) .\end{cases}
$$

$$
E_{k, 1}(z, w ; 0)=E_{k-4}(z) \cdot \vartheta_{m, 0}(z, w)
$$

\section{THE ORTHOGONAL COMPLEMENT OF THE CUSP FORMS}

The Petersson inner product for $f, g \in J_{k, m}(0)$, where at least one of $f$ and $g$ is a cusp form, was already introduced in [6],

$$
\langle f, g\rangle:=\int_{\mathscr{F}} f(z, w) \overline{g(z, w)} y^{k} e^{-4 \pi m N(v) / y} d \omega,
$$

where $w=u+i v$ and $d \omega=y^{-10} d x d y d u d v$ is the invariant volume element. We use the standard identification of $\mathscr{C}_{\mathbb{R}}$ with $\mathbb{R}^{8}$ for normalizing $d u, d v$. 
Lemma 1. Let $k, m \in \mathbb{N}, k>10, q \in a$ with $N(q) \equiv 0(\bmod m)$ and $f \in J_{k, m}^{0}$. Then one has

$$
\left\langle E_{k, m}(z, w ; q), f(z, w)\right\rangle=0 .
$$

Proof. We can write the Eisenstein series in the form (16). Hence the usual unfolding trick gives

$$
\left\langle E_{k, m}(z, w ; q), f(z, w)\right\rangle=\int_{\mathscr{D}} \overline{f(z, w)} y^{k-10} e^{-4 \pi m N(v) / y} d x d y d u d v
$$

where

$$
\mathscr{D}=\left\{(z, w) \in H \times \mathscr{C}_{\mathbb{C}} \mid 0 \leq x \leq 1, u=\sum_{j=0}^{7} u_{j} \alpha_{j}, 0 \leq u_{j} \leq 1\right\}
$$

is a fundamental domain with respect to the translations in (j.1) and (j.2). If one inserts the Fourier expansion (j.4), the integration over $x$ already shows that the integral vanishes.

Next we count the number of possible $q$. Therefore let $\varphi$ denote Euler's totient function.

Lemma 2. Given $m \in \mathbb{N}$ one has

$$
\#\{q: a / m a \mid N(q) \equiv 0(\bmod m)\}=m^{7} \sum_{d \mid m} \frac{\varphi(d)}{d^{4}} .
$$

Proof. Both sides of (21) are multiplicative arithmetical functions. Hence it suffices to consider $m=p^{r}$ for some prime $p$ and $r \in \mathbb{N}$. Due to the corollary in $\S 2$ of [9] the left-hand side of $(21)$ is equal to

$$
p^{4 r}\left[\sum_{\tau=0}^{r} p^{3 \tau}-\sum_{\tau=0}^{r-1} p^{3 \tau-1}\right]=p^{7 r}\left[1+\sum_{\tau=1}^{r}(p-1) p^{-3 \tau-1}\right]=m^{7} \sum_{d \mid m} \frac{\varphi(d)}{d^{4}} .
$$

In particular the number $s$ in (20) can be computed to be

$$
\#\left\{q: a / \operatorname{mo} \mid N(q) \equiv 0(\bmod m), 2 q \in \operatorname{mo}_{\circ}\right\}=: N_{m},
$$

where

$$
N_{m}=\left\{\begin{aligned}
1 & \text { if } m \equiv 1(\bmod 2) \\
136 & \text { if } m \equiv 2(\bmod 4) \\
256 & \text { if } m \equiv 0(\bmod 4)
\end{aligned}\right.
$$

Denote the orthogonal complement of the space of Jacobi cusp forms by

$$
J_{k, m}^{0, \perp}(a)=\left\{f \in J_{k, m}(a) \mid\langle f, g\rangle=0 \text { for all } g \in J_{k, m}^{0}(a)\right\} .
$$

Theorem 3. Let $k, m \in \mathbb{N}, k>10$. Then the set of Jacobi-Eisenstein series $\mathscr{E}_{k, m}$ in (20) forms a basis of $J_{k, m}^{0, \perp}(a)$. Moreover one has

$$
\operatorname{dim} J_{k, m}^{0, \perp}(a)=\frac{1}{2}\left(m^{7} \sum_{d \mid m} \frac{\varphi(d)}{d^{4}}+(-1)^{k} N_{m}\right)
$$

where $N_{m}$ is given by (22). 
Proof. Apply Lemmas 1 and 2, Remark 1 and (17). Given $g \in J_{k, m}(a)$, we conclude from Theorem 2 and (15) that

$$
\begin{aligned}
g- & 2 \sum_{j=1}^{r} \alpha_{g}\left(N\left(q_{j}\right) / m, q_{j}\right) E_{k, m}\left(\cdot, \cdot ; q_{j}\right) \\
& -\frac{1}{2}\left(1+(-1)^{k}\right) \sum_{j=r+1}^{r+s} \alpha_{g}\left(N\left(q_{j}\right) / m, q_{j}\right) E_{k, m}\left(\cdot, \cdot ; q_{j}\right)
\end{aligned}
$$

is a cusp form. Hence the claim follows.

\section{The Selberg trace formula}

Given a subset $S$ of $\Gamma$ and $z, z^{\prime} \in H$ we define

$$
H_{k}^{S}\left(z, z^{\prime}\right):=\sum_{M=\left(\begin{array}{c}
\alpha \\
\gamma
\end{array}\right) \in S}\left(\frac{1}{2 i}\left(z-M \bar{z}^{\prime}\right)\left(\gamma \bar{z}^{\prime}+\delta\right)\right)^{-k}
$$

for $k>2$, where $M z=\frac{\alpha z+\beta}{\gamma z+\delta}$. Then it is well known (cf. [7, 11]) that

$$
\delta_{m} \Phi(z)=\frac{k-1}{4 \pi} \int_{\Gamma[m] \backslash H} y^{\prime k} H_{k}^{\Gamma[m]}\left(z, z^{\prime}\right) \Phi\left(z^{\prime}\right) d \mu\left(z^{\prime}\right)
$$

holds for all $\Phi \in S_{k}(\Gamma[m])$, when $\Gamma[m] \backslash H$ denotes a fundamental domain of $H$ with respect to $\Gamma[m]$ and $d \mu(z)=y^{-2} d x d y$ stands for the invariant volume element. Clearly $\delta_{m}=1$ holds for $m>2$ and $\delta_{1}=\delta_{2}=2$.

Lemma 3. Given $k>6$ and $m \in \mathbb{N}$ one has for all $f \in J_{k, m}^{0}(a)$,

$$
F(z)=\frac{k-5}{8 \pi} \int_{\Gamma \backslash H} y^{\prime k-4} \sum_{M: \Gamma[m] \backslash \Gamma} H_{k-4}^{\Gamma[m] M}\left(z, z^{\prime}\right) \psi(M) \cdot F\left(z^{\prime}\right) d \mu\left(z^{\prime}\right) .
$$

Proof. Let $\mathbb{F}$ be a fundamental domain with respect to $\Gamma$ and $\Gamma=\dot{U}_{j=1}^{r} \Gamma[m] M_{j}$. Then $\bigcup_{j=1}^{r} M_{j} \mathbb{F}$ covers a fundamental domain of $H$ exactly $\frac{2}{\delta m}$-times. Thus Theorem 1 and (23) yield

$$
\begin{aligned}
F(z) & =\frac{k-5}{8 \pi} \sum_{j=1}^{r} \int_{M_{j} \mathbb{F}} y^{\prime k-4} H_{k-4}^{\Gamma[m]}\left(z, z^{\prime}\right) F\left(z^{\prime}\right) d \mu\left(z^{\prime}\right) \\
& =\frac{k-5}{8 \pi} \int_{\mathbb{F}} y^{\prime k-4} \sum_{j=1}^{r} H_{k-4}^{\Gamma[m] M_{j}}\left(z, z^{\prime}\right) \psi\left(M_{j}\right) \cdot F\left(z^{\prime}\right) d \mu\left(z^{\prime}\right) .
\end{aligned}
$$

In the next step we obtain

Lemma 4. Given $k>6$ and $m \in \mathbb{N}$ one has for all $f \in J_{k, m}^{0}(a)$,

$$
\begin{gathered}
f(z, w)=2^{10} m^{4} \frac{(k-5)}{\pi} \int_{\mathscr{F}} y^{\prime k} e^{-4 \pi m N\left(v^{\prime}\right) / y^{\prime}} \sum_{M: \Gamma[m] \backslash \Gamma} H_{k-4}^{\Gamma[m] M}\left(z, z^{\prime}\right) \\
\cdot \Theta(z, w)^{t} \cdot \psi(M) \cdot \overline{\Theta\left(z^{\prime}, w^{\prime}\right)} f\left(z^{\prime}, w^{\prime}\right) d \omega\left(z^{\prime}, w^{\prime}\right) .
\end{gathered}
$$


Proof. Note that the set of all $(z, w)$, where $z \in \mathbb{F}$ and $w$ runs through a fundamental parallelotope of $\mathscr{C}_{\mathbb{C}} / a z+o$ contains 2 copies of a fundamental domain. Write $f=F^{t} \cdot \Theta=\Theta^{t} \cdot F$. The standard procedure yields

$$
\begin{aligned}
& \int_{\mathscr{C}_{\mathrm{c}} / o z^{\prime}+o} \vartheta_{m, q_{j}}\left(z^{\prime}, w^{\prime}\right) \cdot \overline{\vartheta_{m, q_{l}}\left(z^{\prime}, w^{\prime}\right)} e^{-4 \pi m N\left(v^{\prime}\right) / y^{\prime}} d u^{\prime} d v^{\prime} \\
& = \begin{cases}\left(\frac{y^{\prime}}{8 m}\right)^{4} & \text { if } j=l, \\
0 & \text { if } j \neq l .\end{cases}
\end{aligned}
$$

Hence the integral on the right-hand side of (24) equals

$$
\frac{k-5}{8 \pi} \int_{\mathbb{F}} y^{\prime k-4} \sum_{M: \Gamma[m] \backslash \Gamma} H_{k-4}^{\Gamma[m] M}\left(z, z^{\prime}\right) \Theta(z, w)^{t} \cdot \psi(M) \cdot F\left(z^{\prime}\right) d \mu\left(z^{\prime}\right) .
$$

Due to Lemma 3 and $f=\Theta^{t} \cdot F$, the claim follows.

Hence we have computed the Bergmann kernel of $J_{k, m}^{0}(a)$. Thus the standard procedure (cf. $[11,6.4 .1])$ yields the Selberg trace formula. The orthogonal relation (25) then leads to

Theorem 4. Given $k>6$ and $m \in \mathbb{N}$ one has

$$
\operatorname{dim} J_{k, m}^{0}(a)=\frac{k-5}{8 \pi} \int_{\Gamma \backslash H} y^{k-4} \sum_{M: \Gamma[m] \backslash \Gamma} H_{k-4}^{\Gamma[m] M}(z, z)(\operatorname{trace} \psi(M)) d \mu(z) .
$$

Now we are going to calculate the terms explicitly. Note that trace $\psi(M)$ is constant on the conjugacy class of $M$ due to Theorem 1. It is well known (cf. [7, Chapter II]) that the conjugacy classes of hyperbolic elements do not give any contribution.

(A) Contribution from $\pm I, I=\left(\begin{array}{ll}1 & 0 \\ 0 & 1\end{array}\right)$. One has trace $\psi(I)=m^{8}$ and trace $\psi(-I)=$ g.c.d. $(m, 2)^{8}$. Hence the contribution is

$$
\frac{k-5}{24}\left(m^{8}+(-1)^{k} \text { g.c.d. }(m, 2)^{8}\right) \text {. }
$$

(B) Contribution from elliptic elements. There are 6 conjugacy classes of elliptic elements in $\Gamma$, which can be represented by $\pm J, \pm T J, \pm T^{-1} J$. We apply (11) and (12) and evaluate the arising Gauss sums in the usual way in order to get

$$
\begin{gathered}
\operatorname{trace} \psi(J)=\operatorname{trace} \psi(-J)=\text { g.c.d. }(m, 2)^{4} \\
\operatorname{trace} \psi(T J)=\operatorname{trace} \psi\left(-T^{-1} J\right)=1, \\
\operatorname{trace} \psi\left(T^{-1} J\right)=\operatorname{trace} \psi(-T J)=\text { g.c.d. }(m, 3)^{4} .
\end{gathered}
$$

The integrals are evaluated according to [7, Chapter II]. Setting $\rho=\frac{1}{2}(1+i \sqrt{3})$ the total contribution is

$$
\begin{aligned}
& \frac{1}{8} i^{-k} \text { g.c.d. }(m, 2)^{4}\left(1+(-1)^{k}\right)+\frac{1}{6} \frac{\rho^{5-k}}{i \sqrt{3}}\left(1+(-1)^{k} \text { g.c.d. }(m, 3)^{4}\right) \\
& \left.\quad+\frac{1}{6} \frac{\rho^{10-2 k}}{i \sqrt{3}} \text { (g.c.d. }(m, 3)^{4}+(-1)^{k}\right) \\
& \left.=\frac{1}{8} i^{-k} \text { g.c.d. }(m, 2)^{4}\left(1+(-1)^{k}\right)-\left(\frac{k+1}{3}\right) \frac{1}{6} \text { (g.c.d. }(m, 3)^{4}+(-1)^{k}\right),
\end{aligned}
$$

where $\left(\begin{array}{c}k+1 \\ 3\end{array}\right)$ denotes the Legendre symbol. 
(C) Cusp contributions. Representatives of the conjugacy classes of the parabolic elements are given by $\pm T^{j}, j \in \mathbb{Z}, j \neq 0$. Note that

$$
\psi\left(T^{j}\right)=\psi\left(T^{j^{\prime}}\right), \quad \psi\left(-T^{j}\right)=\psi\left(-T^{j^{\prime}}\right), \quad \text { if } j \equiv j^{\prime}(\bmod m) .
$$

According to [7, Chapter II], the integral over all the conjugacy classes $\pm T^{j^{\prime}}, j^{\prime}$ $\equiv j(\bmod m), j=1, \ldots, m$, is evaluated to be

$$
-( \pm 1)^{k} \frac{1}{4 m} \operatorname{trace} \psi\left( \pm T^{j}\right) \text {. }
$$

Due to (11) the total contribution is

$$
\begin{aligned}
-\frac{1}{4 m} \sum_{j=1}^{m}\left(\sum_{q: o / m o} e^{-2 \pi i j N(q) / m}+(-1)^{k} \sum_{\substack{q: o / m_{o} \\
2 q \in m_{o}}} e^{-2 \pi i j N(q) / m}\right) \\
=-\frac{1}{4}(\#\{q: a / m a \mid N(q) \equiv 0(\bmod m)\} \\
\left.\quad+(-1)^{k} \#\left\{q: o / \operatorname{mo|} N(q) \equiv 0(\bmod m), 2 q \in m_{o}\right\}\right) \\
=-\frac{1}{4}\left(m^{7} \sum_{d \mid m} \frac{\varphi(d)}{d^{4}}+(-1)^{k} N_{m}\right),
\end{aligned}
$$

if we regard Lemma 2 and (22).

Gathering all the contributions we obtain our final

Theorem 5. Let $k>6$ and $m \in \mathbb{N}$. Then one has

$$
\begin{array}{r}
\operatorname{dim} J_{k, m}^{0}(a)=\frac{k-5}{24}\left(m^{8}+(-1)^{k} \text { g.c.d. }(m, 2)^{8}\right)+\frac{1}{8} i^{-k} \text { g.c.d. }(m, 2)^{4}\left(1+(-1)^{k}\right) \\
-\left(\frac{k+1}{3}\right) \frac{1}{6}\left(\text { g.c.d. }(m, 3)^{4}+(-1)^{k}\right)-\frac{1}{4}\left(m^{7} \sum_{d \mid m} \frac{\varphi(d)}{d^{4}}+(-1)^{k} N_{m}\right) .
\end{array}
$$

Combining Theorems 5 and 2 we also have an explicit formula for $\operatorname{dim} J_{k, m}(\theta)$.

Corollary 3. Let $k>10$ and $m \in \mathbb{N}$. Then one has

$$
\begin{gathered}
\operatorname{dim} J_{k, m}(a)=\frac{k-5}{24}\left(m^{8}+(-1)^{k} \text { g.c.d. }(m, 2)^{8}\right)+\frac{1}{8} i^{-k} \text { g.c.d. }(m, 2)^{4}\left(1+(-1)^{k}\right) \\
\left.-\left(\frac{k+1}{3}\right) \frac{1}{6} \text { (g.c.d. }(m, 3)^{4}+(-1)^{k}\right)+\frac{1}{4}\left(m^{7} \sum_{d \mid m} \frac{\varphi(d)}{d^{4}}+(-1)^{k} N_{m}\right) .
\end{gathered}
$$

\section{REFERENCES}

1. W. L. Baily Jr., An exceptional arithmetic group and its Eisenstein series, Ann. of Math. (2) 91 (1970), 512-545.

2. H. S. M. Coxeter, Integral Cayley numbers, Duke Math. J. 13 (1946), 567-578.

3. H.-D. Ebbinghaus et al., Numbers, Graduate Texts in Math., vol. 123, Springer-Verlag, New York, Berlin, and Heidelberg, 1990.

4. M. Eichler and D. Zagier, The theory of Jacobi forms, Progr. Math., 55, Birkhäuser, Boston and Basel, 1985. 
5. M. Eie, The Maass space for Cayley numbers, Math. Z. 207 (1991), 645-655.

6. M. Eie and A. Krieg, The Maass space on half-plane of Cayley numbers of degree two, Math. Z. 210 (1992), 113-128.

7. E. Freitag, Hilbert modular forms, Springer-Verlag, Berlin, Heidelberg, and New York, 1990.

8. V. A. Gritsenko, Fourier-Jacobi functions of $n$, J. Soviet Math. 53 (1991), 243-252.

9. M. L. Karel, Fourier coefficients of certain Eisenstein series, Ann. of Math. (2) 99 (1974), 176-202.

10. A. Krieg, Modular forms on half-spaces of quaternions, Lecture Notes in Math., vol. 1143, Springer-Verlag, Berlin, Heidelberg, and New York, 1985.

11. T. Miyake, Modular forms, Springer-Verlag, Berlin, Heidelberg, and New York, 1989.

12. I. I. Pyatetskii-Shapiro, Automorphic functions and the geometry of classical domains, Gordon and Breach, New York, 1969.

13. T. Sugano, On Maass spaces for $S U(2,2)$, Res. Inst. Math. Sci. Kokyuroku 546 (1985), 1-16. (Japanese)

14. C. Ziegler, Jacobi forms of higher degree, Abh. Math. Sem. Univ. Hamburg 59 (1989), 191-224.

Department of Mathematics, National Chung Cheng University, Ming-Hsiung, ChiaYi, Taiwan 621, Republic of China

E-mail address: mkeie@math.ccu.edu.tw

Mathematisches Institut, Westfälische Wilhelms-Universität, Einsteinstrasse 62 , W-4400 MÜNSTER, GERMANY

E-mail address: aloys@math.uni-muenster.de 